\title{
Orientation of letters and errors in their recognition
}

\author{
PAUL A. KOLERS ${ }^{2}$ AND DAVID N. PERKINS \\ RESEARCH LABORATORY OF ELECTRONICS, MASSACHUSETTS INSTITUTE OF TECHNOLOGY
}

\begin{abstract}
Subjects named alphabetic characters that had been rotated, reflected, or inverted. Inversions induce more errors than mirror reflections and reflections induce more than rotations. In a significant number of mistakes a transformed character was assumed to be normally oriented, but in most a transformed character was confused with the mirror image of the original. The data suggest the existence of an "orientation set" in which the identification of ambiguous characters depends largely on their anticipated orientation. The individuality of the data for the different orientations suggests that different transformations are compensated for in part by different mechanisms.
\end{abstract}

Recognizing familiar objects in a variety of orientations is the classic characteristic of object constancy. Little information exists, however, as to whether some orientations are coped with more easily than others and as to whether the coping is all part of a single strategem of object recognition (for example, stimulus generalization). Confusions among geometrically transformed objects are reported both for humans and for other animals, and various sources suggest that some orientations may be more difficult to decode than others (e.g., Sutherland, undated). An experiment in which Ss were required to name inverted, rotated, and reflected alphabetic characters was undertaken in part to study some characteristics of the recognizing of geometrically transformed flat figures.

Many flat figures have a preferred orientation (Ghent, 1961). But most, like the letters kay and ar, are easily recognized in any orientation. Certain figures, however, are perceived and named differently depending on their orientation. The letters bee, dee, pee, and kew are examples. A person must know what the orientation of the letter is in order to name any one of these four correctly with more than chance frequency.

An interaction between orientation and identity is not restricted to letters. Some flat figures, such as the "wife-mother-in-law figure," face in different directions in taking on alternate identities. A silhouette of a dog may become a silhouette of a chef as the figure is rotated (Wallach \& Austin, 1954). In other cases, orientation and identity seem to be independent of each other. Consider Fig. 1, a drawing of a lamp. Turned upside down, the figure can be seen as a bird, tail held high and beak down, pulling a worm out of a box of earth. The bird figure, however, can also be seen as a lamp upside down, and with a little practice the two perceptions can be made to alternate even though the stimulus figure is kept immobile. Mach (1886, trans. 1959, p. 143) has discussed a similar kind of alternation of perception which occurs as the perceiver voluntarily changes only his frame of reference.

The lamp-bird figure and others of its kind show that physical orientation of a figure plays an important but not determining role in perceived orientation. This raises an important issue. When the dual identities and orientations of a figure, such as the lamp-bird figure, are known, often either version can be seen voluntarily. But is the figure perceived upside down because the visual system expects to see a bird, or is the bird perceived because the visual system expects to see something upside down? Prerequisite to the successful identification of some transformed alphabetical characters is a knowledge of their orientation, as pointed out above. In this

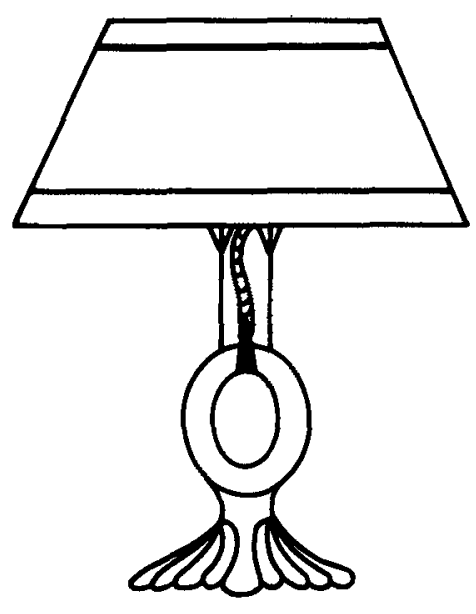

Fig. 1. An ambiguous figure that changes its identity when its orientation is changed. Orientation however may be changed only implicitly, as by imagination, to effect the change in identity.

experiment we examine some of these aspects of the interplay of orientation and identity and show that a figure's orientation may be anticipated before its identity is known (an "orientation set"), and that errors of anticipation occur in ways specific to different orientations.

\section{METHOD}

A computer randomized a page of connected English discourse into 16 different sequences. One set of eight arrangements, called "Letters," preserved only the relative frequency of the letters on the original page. The second set of eight, "Pseudowords," preserved both the relative frequency of the letters and the sequence of word lengths. The eight sequences of Letters and the eight sequences of Pseudowords were transformed geometrically in the manner illustrated earlier (Kolers, 1968), one sequence of Letters and one of Pseudowords in each transformation. Four samples of Letters and four of Pseudowords are illustrated in Fig. 2. N, R, I, and $M$ are simple rotations in three-dimensional space, referred to as normal, rotation, inversion, and mirror reflection. The transformations $\mathrm{rN}, \mathrm{rR}, \mathrm{rl}$, and $\mathrm{rM}$ repeat the simple rotations and in addition rotate every letter on a vertical axis through itself. $^{3}$ Four transformations were named from left to right and the other four from right to left. The asterisk next to each transformation in Fig. 2 shows where it begins.

Each page of material appeared in a single transformation and contained 26 lines. In Letters, there were exactly 32 letters to the line, each letter followed by a blank space, or 832 letters to the page. In Pseudowords there were about 1170 letters to the page, the number of letters varying slightly from line to line.

Ten right-dominant male undergraduates, native speakers of English and familiar only with European (that is, right-going) languages named the letters aloud. Ss were instructed to work as rapidly and as accurately as they could. They were tested individually in two sessions. Half the Ss named the letters of Letters on the first test day and the letters of Pseudowords on the second; the other half worked in the reverse order. The order in which each $S$ received each set of eight 


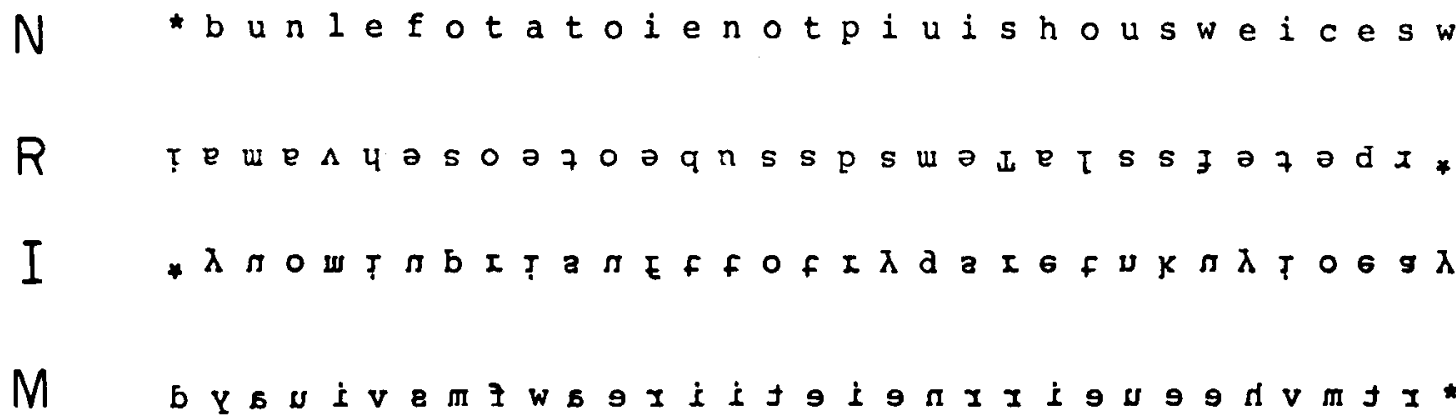

The 1 L

in

(1)

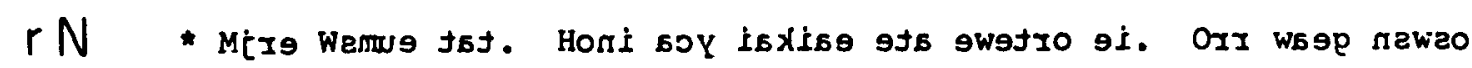

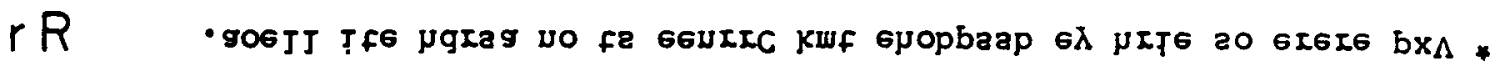

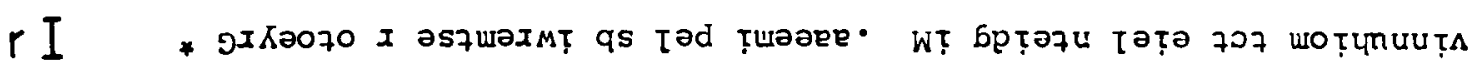

$r M$ itsfcrmbe iryouhhif aan you rasb eeo uuwncddr si pf gssue tgigk *
\end{abstract}

Fig. 2. Eight instances of geometrically transformed letters, the upper four shown as they appeared in Letters and the lower four as in Pseudowords. The asterisks mark the beginning of each line.

transformations was partially counterbalanced to offset the effects of practice. The amount of time taken and the errors the Ss made were recorded. In this paper we are concerned only with the errors and with their implication for understanding the decoding of geometrically transformed flat figures. In another paper we discuss some other aspects of this performance (Kolers \& Perkins, 1969).

\section{Distribution of Errors}

\section{RESULTS}

Altogether, 66,560 letters were named in Letters and about 93,600 in Pseudowords. Although error rates were low, the number of letters named is so large that even low rates yield significant information. Very few errors, 23 in all, were made on normally-oriented letters named from left to right ( $\mathrm{N}$ in Fig. 2). The following analysis therefore is restricted to the seven pages of transformed letters in Letters and in Pseudowords.

The Ss made 2506 errors on the seven pages of Letters and 3894 errors on the seven pages of Pseudowords, rates of 3.8 and $4.2 \%$. Errors are not distributed equally about the alphabet and their frequency varies somewhat with transformation. Table 1 lists the 11 letters on which the greatest number of errors was made, their percentage frequency in the test pages, and the percentage of errors made on them. The column labelled "Total," however, lists the frequencies (e.g., $7 \times 832=5824$ ). The 11 letters comprise $42 \%$ of all the letters that appeared in the test pages but account for about $91 \%$ of all the naming errors. Even within this subset of 11 letters errors are not distributed haphazardly nor according to frequency of appearance of the letters. The letter ef appeared $2 \%$ of the time and was misidentified on $2 \%$ of its appearances in Letters, but the letter bee, which appeared only slightly more frequently, was misnamed $22 \%$ of the time it appeared. The table shows that the four letters bee, dee, pee, kew account for a substantial portion of all errors. These errors are analyzed further in a later section of the paper.

\section{Basis of Errors}

It is useful to know whether the errors are attributable to auditory or phonetic confusions of their names or to difficulties in their visual processing. The letters bee, dee, pee look alike and also have similar-sounding names, and so cannot be used to decide the matter. The problem can be solved by examining a graphical confusion matrix for the 11 letters. Such a matrix is shown in Fig. 3. The upper half of the figure is for Letters, the lower half is for Pseudowords; the left half is for transformations $\mathrm{R}, \mathrm{I}$, and $\mathrm{M}$, and the right half for $\mathrm{rN}, \mathrm{rR}$, $\mathrm{rI}$, and $\mathrm{rM}$. The lines of the graph show the percentage of times it appeared that some letter was called by the name of another (the vertical scale increasing downward). For example, when Pseudowords appeared in Transformation I (lower-left quadrant of the graph), $44 \%$ of the time the letter bee appeared it was called "dee"; and when Letters appeared in Transformation $\mathrm{rl}$ (the upper-right quadrant of the graph), $18 \%$ of the time the letter en appeared it was called "yoo."

The figure answers the question of visual or phonetic confusion by showing that the errors cluster into subsets whose members are sensitive to visual confusion. The names en-yoo, ef-tee, and ay-ess do not sound alike but these letters were confused. Moreover, while bee-dee-pee have similar sounding names, the letter kew, which was confused with them, does not sound like them; and tee, whose name does sound like theirs, was never confused with them. The errors are clearly due not to phonetic or memorial confusion of their names but to confusions in visual processing.

A close inspection of Fig. 3 reveals that the frequency of errors varies somewhat with the letter being named and with the transformation imposed. In general, transformations I and $\mathrm{rR}$ induce many confusions, especially among the letters bee-dee-pee-kew; the letters ay and ess are especially affected by transformations $\mathrm{M}$ and $\mathrm{rN}$, and so on. A clearer indication of the effects of orientation can be obtained from a close examination of the confusions among the four characters bee, dee, pee, and kew. 
Table 1

Frequency of Letters and of Errors (Percent)

\begin{tabular}{|c|c|c|c|c|c|c|c|c|c|c|c|c|}
\hline \multicolumn{13}{|c|}{ Letter Named } \\
\hline Letters & a & $\mathrm{b}$ & d & $\mathrm{f}$ & $\mathrm{g}$ & $\mathrm{n}$ & $\mathrm{p}$ & $q$ & s & $\mathrm{t}$ & $\mathrm{u}$ & Total \\
\hline Distribution in pages & 7 & 2.4 & 3 & 2 & 2 & 5.9 & 1.8 & 0.1 & 6.3 & 7.6 & 3.3 & $2425 / 5824=42 \%$ \\
\hline Errors & 4.6 & 22 & 19 & 2 & 10 & 12 & 25 & 52 & 4 & 6 & 7 & $2274 / 2506=91 \%$ \\
\hline \multicolumn{13}{|l|}{ Pseudowords } \\
\hline Distribution in pages & 7.2 & 2.2 & 3.2 & 1.9 & 2.3 & 5.7 & 1.8 & 0.1 & 6.4 & 7.8 & 3.1 & $3420 / 8190=42 \%$ \\
\hline Errors & 8.5 & 33 & 30 & 2.9 & 8.8 & 9.2 & 25 & 51 & 4.9 & 2.9 & 7 & $3535 / 3894=91 \%$ \\
\hline
\end{tabular}

\section{Errors of Orientation}

All of the letters on a single page appeared in the same transformation. If orientation of the page were assessed only once by $S$ he would always call a given letter by the same name wherever it appeared on that page. Errors occurred when $S$ in fact called a given character by different names on its various appearances in a single page. A tabular confusion matrix for the errors of naming $b, d, p, q$, Table 2 , illuminates Ss' ability to cope with various transformations.

The left-most column of the table lists the transformations that were imposed on the letters. The columns headed "Letter" and "Resembles" indicate the letter S was naming and the letter it resembles when transformed. The major cells of the table show the percentage of times the transformed letter was called by the names of the letters in the cells.
Consider the major row for physical transformation R. When " $b$ " is rotated it resembles " $q$ " and Ss called rotated bees "kew" 2\% of the time in Pseudowords and 4\% of the time in Letters (column headings $\mathrm{P}$ and $\mathrm{L}$ ). However they called rotated bees "pee" $1 \%$ of the time in Pseudowords, and called them "dee" 28\% of the time in Pseudowords and 15\% of the time in Letters. We contend that transformed letters are named according to $S$ 's assumption about their orientation (not necessarily a conscious assumption) and that the error $\mathrm{S}$ makes reveals his surmise. These assumptions are indicated under "Type of Error (Implicit Transformation)" and require a word of explanation.

The $S$ can exercise either of two options in naming a transformed letter. He can assume that the page and the character are normally oriented--an identity operation-and
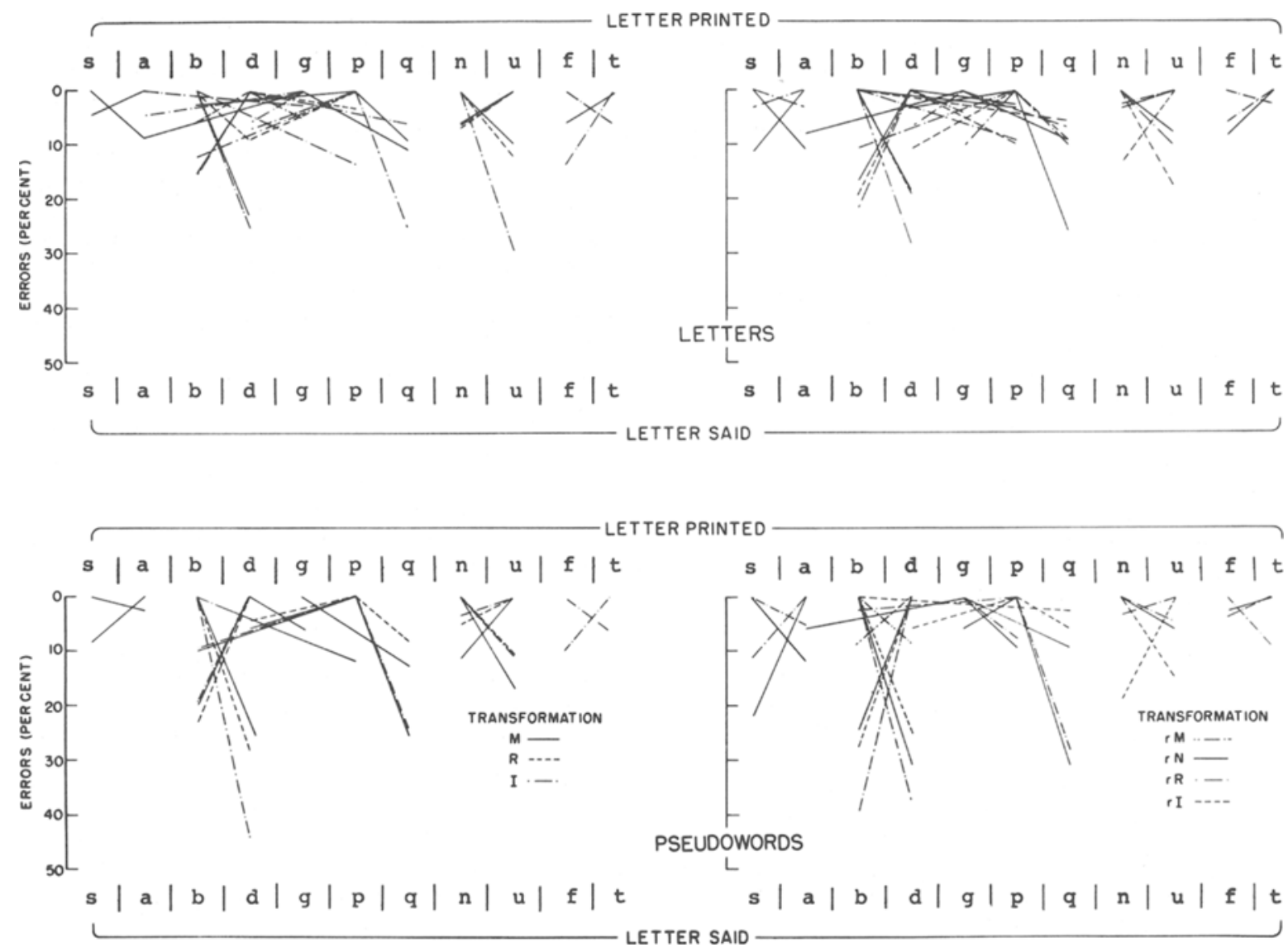

Fig. 3. Confusion matrix of transformed letters. The lines indicate the percentage of times (increasing downward) that a printed letter was called by the name of another. 
Table 2

Confusion Errors in Naming Transformed Letters (Percent)

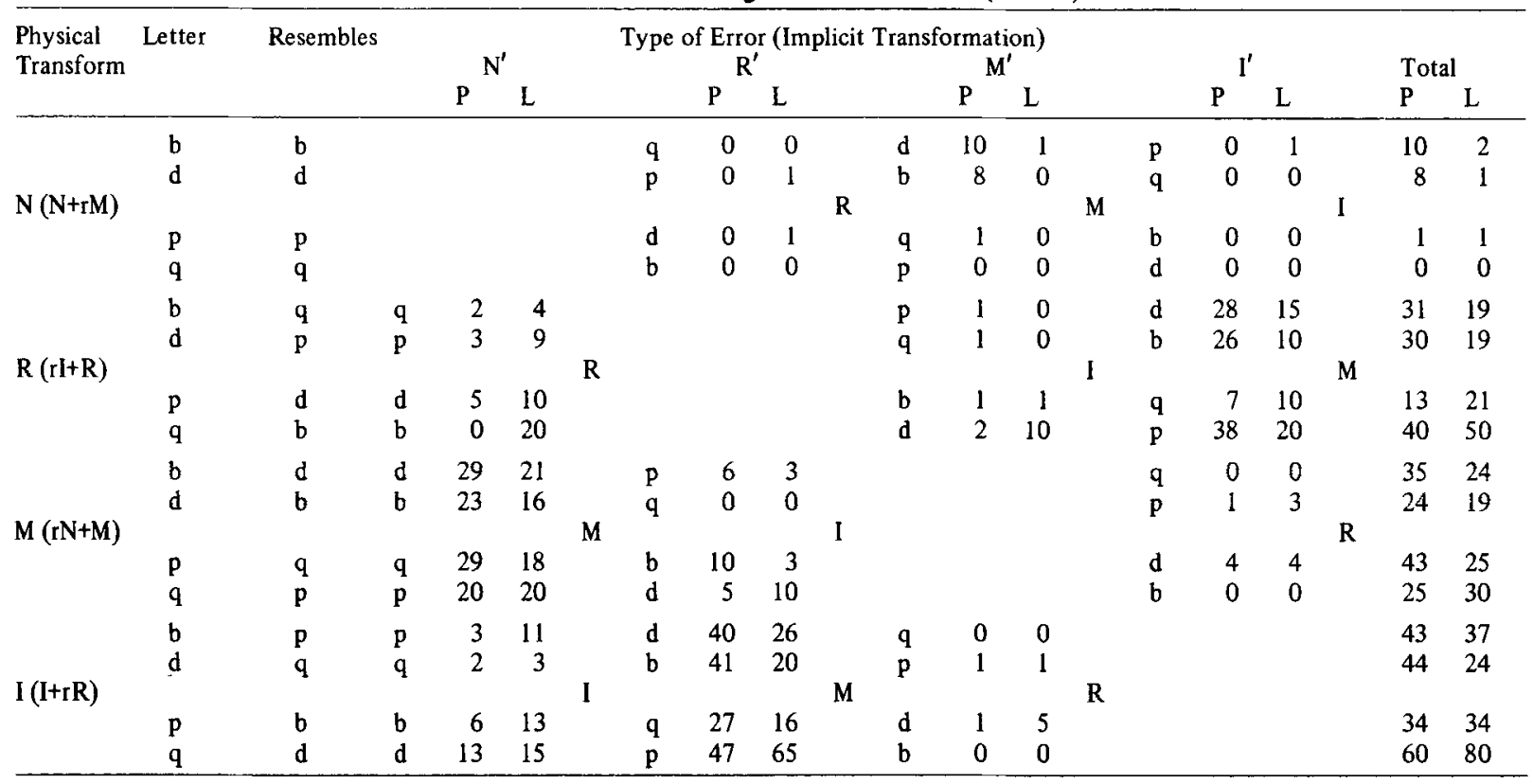

call a letter what it looks like under those conditions (the column indicating Resemblance in Table 2). In other cases of error he appreciates that the characters have been transformed but makes an incorrect assumption about the transformation. ${ }^{4}$ He has a transformation in mind, but the wrong one. Thus if $\mathrm{S}$ sees "b" and says "pee," one may guess that he had inversion in mind as the transform. This reasoning allows us to infer the implicit transformations applied by $S$ when he made errors. These implicit transformations that guided the incorrect naming of the letters are indicated by the letters $N^{\prime}, R^{\prime}, M^{\prime}$, and $\mathrm{I}^{\prime}$ in Table $2 . \mathrm{N}^{\prime}$ is the identity operation; $\mathrm{R}^{\prime}, \mathrm{M}^{\prime}$, and $\mathrm{I}^{\prime}$ are the implicit transformations.

The capital letter in each cell of Table 2 describes the geometric relation between the transformation actually imposed on the text and the transformation implicit in S's response. For example, when the physical transformation was $R$ and the implicit transformation was $I^{\prime}$ the cell contains $M$, because rotation and inversion are mirror reflections of one another, i.e., $R=M(I)$ and $I=M(R)$. (In stating this relation only individual characters are considered and direction of naming is ignored: both $\mathrm{rI}$ and $\mathrm{R}$ are treated as $\mathrm{R}, \mathrm{I}$ and $\mathrm{rR}$ are treated as I, etc.) The final columns under "Total," sum the errors for each transformation. ${ }^{5}$

Table 2 yields three kinds of information: (1) the potency of the transformations to induce errors; (2) Ss' preferred implicit assumptions in compensating for transformations; and (3) the geometrical relation between the implicit and the actual transformation.

(1) The last two columns of the table (Total) show that the transformations are not equally difficult. Physical inversion (I and $\mathrm{rR}$ ) induces the greatest percentage of errors, mirror reflection ( $\mathrm{rN}$ and $\mathrm{M}$ ) induces fewer, and rotation in the plane ( $\mathrm{rl}$ and $\mathrm{R}$ ) induces the fewest. Note that it is a transformation that produces difficulties, not merely top-to-bottom orientation. The characters in transformations $\mathrm{R}$ and $\mathrm{rl}$ are upside down and induce some errors; those in $I$ and $r R$ induce many more. Furthermore, the upside-down characters of $\mathrm{R}$ and $\mathrm{rI}$ are misidentified less often than the right-side up characters of $\mathrm{M}$ and $\mathrm{rN}$. (Because of the small sample size, errors on kew may be overestimates; see Fn. 5.)

(2) Incorrect assumptions about orientation do not occur haphazardly. Examination of the major columns of Table 2 shows that a likely incorrect assumption about orientation is that the characters are normal (Column $\mathrm{N}^{\prime}$, the identity operation). However, when $S$ appreciated that the letters had been transformed but mistook the transformation, the most prevalent assumption was that the characters were rotations in the plane; the next most frequent transformation confusion was that the characters were inverted; and least likely was the hypothesis that the characters were mirror reflections. Thus the order of preference in decoding is $N^{\prime}, R^{\prime}, I^{\prime}, M^{\prime}$.

(3) The geometrical relation between the imposed transformation and S's assumption, as expressed by what he said in error, is shown by the capital letter in each cell of Table 2. The largest percentages are associated with the letter M. [The dependence of the implicit transformation upon the imposed transformation can be expressed as the likelihood with which an assumption will be held given a fact. In standard notation $\mathbf{p}\left(R^{\prime} \mid I\right)>p\left(R^{\prime} \mid M\right)$; $p\left(I^{\prime} \mid R\right)>p\left(I^{\prime} \mid M\right)$; etc.] In making errors despite appreciating the fact that characters had been transformed, Ss confuse characters with their mirror images. Mirror reflection is not "difficult," as is sometimes asserted; it is equivocal. Mirror reflection does not induce the greatest number of errors; inversion does that. S, however, often cannot tell the difference between a character and its mirror image even in memory, especially when the characters are upside down.

\section{DISCUSSION}

Our method allows us to separate (1) the impact on $S$ of various two-dimensional orientations from (2) preferred compensatory or coping strategies. With respect to impact, inversion induces the greatest number of errors, followed by mirror reflection, rotation in the plane, and normal $(I, M, R, N)$. The errors are due to visual confusions of shapes and not to auditory confusions of the names of the letters (Fig. 3).

The order of difficulty of the transformations obtained from the frequency of errors is identical with the order obtained from speed of naming (Kolers \& Perkins, 1969); but two points make it clear that frequency of errors is not the basis of the differences in time, nor differences in time the basis of frequency. The first is that only $4 \%$ of responses on the average were errors, as described in the Results under Distribution of Errors, while differences in speed are greater. 
Thus there are too few errors to account wholly for the differences in speed, and too much difference in speed to account for the small number of errors. Secondly, it was pointed out with connected discourse (Kolers, 1968) that the order of difficulty of transformations remains unaffected when all the erroneous responses are removed from the time data. We conclude, therefore, that difficult transformations both induce more errors and take longer to be decoded and that neither of these effects is due wholly to the other.

Most of S's responses are correct (Table 1) but when he is faced with a transformed character he sometimes makes errors; these may be taken as expressions of preferred coping or compensatory strategies. A highly likely error (and thus a preferred strategy) was to assume that a letter was not transformed and to name it as the normal letter it resembled. Otherwise, Ss were likely to name letters as their rotations, inversions, or mirror reflections, in that order. Thus the order of preference for compensation is $N^{\prime}, R^{\prime}, I^{\prime}, M^{\prime}$, shown as the implicit transformations of Table 2. This order is almost the complement of the order of difficulty $(I, M, R, N)$. The relation between the two orders raises the question whether discrete compensatory strategies are applied serially. If they were, the small number of errors induced by $R$ and $N$ might be only the statistical result of success in applying these "most favored" strategies in order.

\section{Orientation Sets}

One of the issues of this experiment was whether "orientation sets" exist. If they do, their existence would be a vote for the significance of orientation not just as a byproduct of perceptual processing, but as an independent factor important in the constructing of perceptions. The data do seem to support the idea. The ambiguous letters bee, dee, pee, and kew are named correctly (that is, in agreement with the orientation of the other letters on a page) with a likelihood much better than chance (Table 2). The Ss, therefore, brought information to their encounter with the ambiguous letters which often allowed them to answer correctly.

Furthermore, when making errors, Ss were much more able to keep track of the up-down aspects of what they were naming than the left-right aspects (the small capital letters within the cells of Table 2). This, too, implicates a specifically geometric polarization in their performance appropriate to an orientation set, but not appropriate to a general and undifferentiated capacity to cope with transformed objects.

It seems clear that implicit assessment of orientation is a separate and continuing aspect of character recognition. If the building of a percept on the basis of an orientation were done only once for a whole page of characters-as by making a "judgment" about orientation or by "adapting"-identical characters would be named the same way on their successive appearances on the same page. This did not occur. Nevertheless, it seems extravagant to assert that orientation is computed anew for every character examined. The lesser variance (the sharper patterning) in Pseudowords than Letters (Fig. 3) suggests that segments larger than individual letters are often treated together.

How much and what sort of information is contained in an orientation set is a major problem. In the patterning of the errors of naming transformed letters two sorts of mistakes dominate (Table 2). The more frequent occurs when the transformation $S$ assumes the character to be in is the mirror reflection of the actual transformation, shown by $\mathrm{M}$ within the cells of Table 2. The other is that the transformed letter is treated as if it were normally oriented (Column $\mathrm{N}^{\prime}$ ). Two quite different mechanisms may underlie these two sources of error. ${ }^{6}$ Mistakes might occur because the orientation set itself was ambiguous, labile, uncertain, or even forgotten, or they might occur because the arrival of the eye on a new character triggered processes which outweighed evidence brought via the set. The latter seems most appropriate to describe S's reverting to a normal interpretation of a transformed character, and the former to describe S's assuming a character to be the mirror reflection of the correct transformation.

\section{Mechanisms of Decoding}

It is misleading, however, to think that the errors are characterized only by a greater capacity to cope with up-down misorientations than left-right misorientations. Rather, each transformation has its own characteristics. For example, when $\mathrm{I}$ is presented it is mistaken for $\mathrm{R}$ more often than $\mathrm{R}$ is named as I (Table 2). This asymmetry shows that the errors involve something more than an uncertain sense of left-right. Furthermore, both $I$ and $r R$ and $M$ and $r N$ induce more errors than $\mathrm{R}$ and $\mathrm{rI}$. This implies that the phenomenal upright is not the decisive variable in these results (cf. Rock, 1966). One conjecture is that $\mathrm{R}$, like $\mathrm{N}$ but in a much weaker way, is a perceptually stable state.

The individual influence of the different transformations (Table 2) is decisive evidence against an alternative proposal that some homogeneous mechanism in the visual systems copes with misorientations. If ay and ess are confusable in some orientation, they should be confused in any orientation if a single mechanism produced the results, and similarly with en-yoo and ef-tee. In fact, however, confusions are orientation specific (Fig. 3 and Table 2).

Even a broader theory than, say, stimulus generalization or adaptation that proposes a single mechanism to account for both the particular orientation and the shape of a letter would fail to match the data. Each transformation has its individual error pattern. We take this as evidence that the recognition of transformed objects is accomplished in part by orientationspecific mechanisms. It is entirely conceivable that there are specific constructive strategies for building percepts of objects in the more common misorientations such as $\mathrm{R}, \mathrm{I}$, and $\mathrm{M}$.

\section{REFERENCES}

GHENT, L. Form and its orientation. American Journal of Psychology, $1961,74,177-190$.

KOLERS, P. A. The recognition of geometrically transformed text Perception \& Psychophvsics. 1968, 3, 57-64.

KOLERS, P. A., \& PERKINS, D. N. Orientation of letters and their speed of recognition. Perception \& Psychophysics, 1969, 5, 275-280. MACH, E. The analysis of sensations. New York: Dover, 1959.

ROCK, 1. The nature of perceptual adaptation. New York: Basic Books, 1966

SUTHERLAND, N. S. Shape discrimination by animals. Exp. Psychol. Soc. Monograph, No. 1, undated.

WALLACH, H., \& AUSTIN, P. Recognition and the localization of visual traces. American Journal of Psychology, 1954, 67, 338-340.

\section{NOTES}

1. This work was supported principally by the National Institutes of Health (Grants 1 PO1 GM-14940-01 and 1 PO1 GM-15006-01) and in part by the Joint Services Electronics Program [Contract DA 28-043AMC-02536(E)]. Some of our suggestions were worked out in Project Zero, Harvard Graduate School of Education. We also thank Saul Sternberg, Ulric Neisser, and Murray Eden for their judicious comments on some of these issues.

2. Address: Bell Telephone Laboratories, Holmdel, New Jersey 07733.

3. We preserve this notation to facilitate comparison with the experiment on reading (Kolers, 1968); for isolated characters it is less apt. The eight transformations actually create only four physical arrangements (Fig. 2); thus, $\mathrm{N}$ and $\mathrm{rM}, \mathrm{R}$ and $\mathrm{rI}, \mathrm{I}$ and $\mathrm{rR}$, and $\mathrm{M}$ and $\mathrm{rN}$ are identical pairs of samples. The pairs differ in the direction in which they were named, not in their geometry.

4. The error is made not in identifying the character but in identifying the transformation. Dee was never confused with eks or jay or el, for example; it was confused with bee, gee, pee, and kew.

5. Kew occurs infrequently in written discourse and so was under-represented in the experiment (Table 1); it appeared only once or twice per page.

6. The percentages in the cell at the conjunction of Row $M$ and Column $\mathrm{N}^{\prime}$ may actually represent the action of two separate mechanisms: The action of the identity operator $\left(N^{\prime}\right)$ and the tendency to confuse things with their mirror images.

(Accepted for publication November 27, 1968.) 